\title{
Índices de Privação Socioeconômica e de Ruralidade: ferramentas para o planejamento e pesquisa em Saúde em nível dos municípios brasileiros*
}

\section{Índices de Privación Socioeconómica y de Ruralidad: herramientas para la planeación y la investigación en salud a nivel de los municipios brasileños}

\section{Socioeconomic Deprivation and Rurality Indicators: Tools for the Planning and Research in Health among the Brazilian Towns}

Recepção: 20 Julho 2018. Aprovação: 04 Junho 2019. Publicação: 29 Novembro 2019.

DOI: https://doi.org/10.11144/Javeriana.rgps18-37.ipsr

\author{
Tamires Carneiro de Oliveira Mendes ${ }^{a}$ \\ Universidade Federal do Rio Grande do Norte , Brasil \\ ORCID: http://orcid.org/0000-0002-7254-4096 \\ Marc Marí-Dell'Olmo \\ Universitat Pompeu Fabra, España \\ ORCID: http://orcid.org/0000-0003-4345-4988 \\ Albert Pratsb Uribe \\ Agència de Salut Pública de Barcelona, España \\ ORCID: http://orcid.org/0000-0003-1202-9153 \\ Kenio Costa Lima \\ Universidade Federal do Rio Grande do Norte, Brasil \\ ORCID: http://orcid.org/0000-0002-5668-4398 \\ André Luiz Barbosa De Lima \\ Universidade Federal do Rio Grande do Norte, Brasil \\ ORCID: http://orcid.org/0000-0002-0030-6638 \\ Carme Borrell \\ CIBER de Epidemiología y Salud Pública, Madrid, España \\ ORCID: http://orcid.org/0000-0002-1170-2505 \\ Maica Rodríguez Sanz \\ Agència de Salut Pública de Barcelona, España \\ ORCID: http://orcid.org/0000-0002-3772-1798
}

\begin{abstract}
Como citar este artigo: Mendes TCO, Marí-Dell'Olmo M, Uribe AP, Lima KC, De Lima ALB, Borrell C, Rodríguez Sanz M. Índices de Privação Socioeconômica e de Ruralidade: ferramentas para o planejamento e pesquisa em Saúde em nível dos municípios brasileiros. Revista Gerencia y Políticas de Salud. 2019;18(37). http s://doi.org/10.11144/Javeriana.rgps18-37.ipsr
\end{abstract}

a Autora correspondente. Correio electrónico: tamires.carneiro@hotmail.com 


\section{Resumo}

O presente estudo ecológico visa construir e disponibilizar índices socioeconômicos dos municípios do Brasil, analisálos espacialmente e compará-los com a Esperança de vida. A partir de dados secundários do ano 2010, construiuse os índices em três etapas: teórica; análise estatística uni e bivariada, e Análise de Componentes Principais; e aplicação dos índices por meio de análise espacial e comparação com a Esperança de vida. Foram selecionadas 13 variáveis, que compuseram dois índices: Privação Socioeconômica (IPS) e Ruralidade (IR), sendo o IPS o de maior relação com a Esperança de vida, dividindo espacialmente o país em dois: uma porção inferior de maior vantagem socioeconômica e uma superior mais vulnerável. A IR complementa a IPS, mostrando que os municípios de maior privação socioeconômica e mais rurais têm menor esperança de vida. Assim, o IPS e o IR destacaram 2149 municípios de maior necessidade de atenção, localizados, especialmente, nas regiões Norte e Nordeste.

Palavras-chave: determinantes sociais da saúde, análise socioeconômica, análise fatorial, disparidades nos níveis de saúde, análise espacial, indicadores básicos de saúde.

\section{Resumen}

El presente estudio ecológico busca construir y facilitar índices socioeconómicos de los municipios de Brasil, analizarlos espacialmente y compararlos con la esperanza de vida. A partir de datos secundarios del año 2010, se construyeron índices en tres etapas: teórica; análisis estadística uni y bivariada, y análisis de componentes principales; y aplicación de los índices por medio de análisis espacial y comparación con la esperanza de vida. Se seleccionaron 13 variables que compusieron dos índices: Privación Socioeconómica (IPS) y Ruralidad (IR), siendo el IPS el de mayor relación con la esperanza de vida, dividiendo espacialmente el país en dos: una porción inferior de mayor ventaja socioeconómica y una superior más vulnerable. La IR complementa la IPS, mostrando que los municipios de mayor privación socioeconómica y más rurales tienen menor esperanza de vida. Así, el IPS y el IR destacaron 2149 municipios de mayor necesidad de atención, localizados, especialmente, en las regiones norte y noreste.

Palabras clave: determinantes sociales de salud, análisis socioeconómico, análisis factorial, disparidades en los niveles de salud, análisis espacial, indicadores básicos de salud.

\section{Abstract}

This ecological study seeks to construct and make available some socioeconomic indicators about the Brazilian towns and, then, to analyze them in detail and compare them to the life expectancy. Based on secondary data from 2010 , the indicators were constructed in three stages: theoretical stage; univariate and bivariate statistical analysis and main component analysis; and application of indicators according to a spatial analysis and comparison to the life expectancy. Thirteen (13) variables were selected to construct two indicators: socioeconomic deprivation indicator (IPS) and rurality indicator (IR), IPS being the higher in relation to the life expectancy. It showed a spatial division of the country in two areas: a lower area with better socioeconomic advantage and a higher area with greater vulnerability. The IR indicator complements the IPS one, thus showing that the towns with a greater socioeconomic deprivation and a higher rurality indicator show a lower life expectancy. This way, the IPS and IR indicators reported 2149 towns with higher care needs, particularly those located in the northern and north-eastern regions.

Keywords: social determinants of health, socioeconomic analysis, factor analysis, healthcare level inequalities, spatial analysis, basic health indicators. 


\section{Introdução}

Iniquidades na distribuição da renda, poder e recursos nos níveis global, nacional e local exercem influência sobre a maior parte da carga das doenças e sobre as desigualdades em saúde, através das condições sociais nas quais as pessoas nascem, crescem, vivem, trabalham e envelhecem, condições estas denominadas Determinantes Sociais da Saúde - DSS $(1,2)$. Tendo em vista que "o gozo do mais alto nível de saúde que se possa atingir constitui um dos direitos fundamentais de todo ser humano, sem distinção de raça, religião, credo político e condição econômica ou social" (3), as iniquidades em saúde são realidades injustas e, em grande parte, evitáveis. Para que as mesmas sejam reduzidas, torna-se necessário medir e compreender o problema, com especial atenção aos determinantes que geram estratificação social, o que ainda representa uma lacuna na região da América Latina $(2,4)$.

Nesse contexto, o território/área onde os indivíduos residem se insere em modelos teóricos de DSS como um eixo de desigualdade, determinando hierarquias de poder na sociedade de modo estrutural (5). Os fatores socioeconômicos relacionados ao território (contextuais) se apresentam com variadas dimensões na literatura a respeito dos modelos de DSS, entre as quais podemos destacar nove de maior importância no contexto brasileiro: Aspectos econômicos; Educação; Trabalho; Habitação; Demografia; Políticas sociais; Segurança pública; Capital social (relações sociais vigentes) e Cultura e valores $(1,5,6,7,8)$.

Para a produção de evidências acerca do nível socioeconômico contextual, os censos e institutos de pesquisas governamentais comumente produzem e disponibilizam um amplo universo de indicadores, que permitem uma compreensão robusta do tema, porém este grande número de variáveis dificulta a execução de análises nas pesquisas em saúde, bem como sua interpretação. Por outro lado, a seleção de apenas algumas variáveis constitui um difícil processo de tomada de decisão acerca daquelas poucas que melhor representam este complexo determinante, ou, ainda, pode ocorrer que a multidimensionalidade do mesmo não seja contemplada satisfatoriamente. Assim, por não haver uma medida simples e direta, medidas compostas vêm sendo construídas com fins de abordar as questões socioeconômicas em um índice resumido e multidimensional, como o Índice de Desenvolvimento Humano - IDH, OECD Better Life Index, que produzem informações para países.

No Brasil, em nível de municípios, tem-se o IDH e o Índice Firjan de Desenvolvimento Municipal - IFDM, por exemplo, que, apesar de traduzirem o grau de desenvolvimento socioeconômico do território, não têm o objetivo de abordar o modelo de DSS e, portanto, apresentam lacunas com relação ao modelo teórico do mesmo. Ademais, os dois índices em questão possuem variáveis de saúde na sua composição, o que não é interessante para o estudo da relação dos fatores socioeconômicos com a saúde, já que esta se apresentaria de forma duplicada na análise, posicionada como variável dependente e inserida na independente, além de provocar uma distorção na medida do perfil socioeconômico. 
Tamires Carneiro de Oliveira Mendes/ Marc Marí-Dell’Olmo/ Albert Pratsb Uribe/ Kenio Costa Lima/ et ai.

Assim, visando contribuir para que os estudos e o planejamento de políticas públicas para a redução das iniquidades em saúde sejam mais congruentes com a realidade e, portanto, mais eficazes, o presente estudo tem o objetivo de construir medidas compostas que resumam o universo de indicadores do nível socioeconômico contextual para os municípios do Brasil, no ano 2010, com base nos marcos conceituais dos Determinantes Sociais da Saúde, analisá-lo espacialmente e compará-lo com um indicador de saúde: Esperança de vida.

\section{Materiais e métodos}

\section{Tipo e cenário do estudo}

Trata-se de um estudo do tipo transversal e ecológico, que toma o agregado de base territorial como tipo operativo para fazer inferências sobre as condições de vida dos indivíduos, cuja área de investigação diz respeito ao Brasil. Para que o índice traduza de modo mais fidedigno possível o contexto em que vivem os indivíduos, utilizou-se a menor unidade territorial disponível nas fontes estatísticas públicas e oficiais: os 5565 municípios brasileiros existentes em 2010, com número médio de 34.278 habitantes, variando de 805 em Borá/SP a 11.253.503 em São Paulo/ SP (9).

Quanto aos cinco novos municípios fundados em janeiro de 2013 (Pescaria Brava e Balneário Rincão, em Santa Catarina; Mojuí dos Campos, no Pará; Pinto Bandeira, no Rio Grande do Sul e Paraíso das Águas, no Mato Grosso do Sul), os quais constituíam distritos pertencentes a outros municípios, não foi possível analisá-los isoladamente, devido a ausência de dados para o ano de 2010 (10). Portanto, os mesmos foram analisados como parte do município ao qual pertenciam (Laguna, Içara, Santarém, Bento Gonçalves e Água Clara, respectivamente).

\section{Variáveis do estudo e análise dos dados}

As variáveis do estudo representam dados contextuais agregados referentes ao ano do último censo, 2010, coletados a partir de fontes secundárias públicas e oficiais: Instituto de Pesquisa Econômica Aplicada - Ipea (11) e Instituto Brasileiro de Geografia e Estatística - Ibge (10), tanto em sua forma bruta, quanto transformada em indicadores pelo Programa das Nações Unidas para o Desenvolvimento - PNUD (9).

Baseado no trabalho de Carr-Hill e Chalmers-Dixon (12), o resumo do amplo universo de variáveis para a produção dos índices contextuais, se deu em três etapas, a saber: teórica, através da consulta da literatura acerca dos marcos conceituais de DSS e construção das dimensões do nível contextual por um processo de exposição-discussão $(1,5,6,7,8)$; estatística, por meio de análise univariada, bivariada e multivariada pela análise de componentes principais; e aplicação dos índices comparando com um indicador de saúde (Esperança de vida) e por análise espacial, conforme descritas abaixo. 
Dentre as mais de 800 variáveis disponíveis nas fontes públicas de dados, foram selecionadas e analisadas aquelas com maior importância teórica para a análise em saúde em nível de municípios do Brasil, buscando contemplar as dimensões delimitadas na consulta dos marcos conceituais $(1,5,6,7,8)$. A etapa estatística se iniciou pela análise univariada das variáveis selecionadas pelo marco teórico, com fins de identificar aquelas que apresentam um considerável grau de variabilidade na distribuição de seus valores e, portanto, com maior potencial para discriminar os municípios, bem como aquelas com uma distribuição mais próxima da normalidade. Foram avaliados a distribuição dos dados por meio de histograma e medidas estatísticas descritivas (média, desvio-padrão, valores mínimo e máximo, mediana, percentis 25 e 75$)$.

Em seguida, utilizou-se análise bivariada por meio da correlação de Spearman entre as variáveis de cada dimensão do nível contextual para selecionar aquelas que têm maior potencial de representar todas as variáveis das respectivas dimensões, permanecendo nas próximas etapas apenas as variáveis que apresentaram maior número de correlações significativas e de maior valor em relação ao todo. Ademais, verificou-se a correlação de todas as variáveis com o indicador de saúde utilizado (Esperança de vida) para identificar aquelas com maior potencial de explicação das iniqüidades em saúde. Optou-se por utilizar uma técnica não-paramétrica, a correlação de Spearman, para que se tenha uma maior validez na interpretação da análise com variáveis que não apresentam distribuição normal dos dados.

Dentre as variáveis que passaram por todas as etapas anteriores, a escolha de uma quantidade mínima para representar cada dimensão dos fatores socioeconômicos utilizou como critérios a qualidade teórica da variável e as mais utilizadas pela literatura, a fim de fornecer maior potencial de comparabilidade com outros estudos. A etapa estatística finalizou com a construção dos índices a partir da Análise de Componentes Principais, na qual as variáveis se agrupam de acordo com as dimensões latentes no seu conjunto e interpretadas por aquilo que representam coletivamente (13).

Previamente a esta análise multivariada, fez-se o exame dos dados a fim de identificar os dados perdidos (ausentes) que poderiam prejudicar o resultado, sendo os mesmos substituídos por dados estimados por intermédio da análise de regressão em relação a todas as variáveis coletadas. Quanto aos municípios outliers, optou-se por mantê-los com seus dados, uma vez que se pretende analisar o grupo de municípios do Brasil por inteiro.

As variáveis incluídas foram testadas a fim de observar se eram suficientemente correlacionadas umas com as outras para produzir componentes representativos. Como método de avaliação, foram utilizados a matriz de correlação, o teste de esferecidade de Bartlett e a medida de adequação da amostra pelo Índice de Kaiser-Meyer-Olkin (KMO) e pela matriz anti-imagem. Com base em tais suposições estatísticas, seguidas do percentual que os componentes explicam de cada variável (comunalidades) e o percentual de variância explicada por todo o modelo, escolheu-se a combinação de variáveis que gerava o modelo mais ajustado e com maior riqueza 
teórica para o seu agrupamento e construção dos índices, sendo selecionadas 13 variáveis, que estão apresentadas no tabela 1.

\section{Tabela 1.Caracterização das variáveis do estudo sobre medida dos indicadores socieconômicos contextuais. Brasil, 2018}

\begin{tabular}{|c|c|c|c|}
\hline Dimensáo & Variável & Definiçấo & Fonte \\
\hline $\begin{array}{l}\text { Aspectos } \\
\text { económicos }\end{array}$ & $\begin{array}{l}\text { Renda per capita média } \\
\text { (Renda) }\end{array}$ & $\begin{array}{l}\text { Razão entre o somatório da renda de todos os individuos residentes } \mathrm{em} \\
\text { domicílios particulares permanentes e o número total desses indivíduos }\end{array}$ & PNUD-Ibge \\
\hline $\begin{array}{l}\text { Aspectos } \\
\text { económicos }\end{array}$ & Indice de Gini (Gini) & $\begin{array}{l}\text { Medida do grau de desigualdade existente na distribuição de individuos } \\
\text { segundo a renda domiciliar per capita. Varia de } 0 \text {, quando não há } \\
\text { desigualdade (a renda domiciliar per capita de todos os indivíduos tem o } \\
\text { mesmo valor), a } 1 \text {, quando a desigualdade é máxima (apenas um } \\
\text { indivíduo detém toda a renda) }\end{array}$ & PNUD-Ibge \\
\hline Educação & $\begin{array}{l}\text { Taxa de analfabetismo- } 18 \text { anos } \\
\text { ou mais (Analf) }\end{array}$ & $\begin{array}{l}\text { Razáo entre a população de } 18 \text { anos ou mais de idade que não sabe ler } \\
\text { nem escrever um bilhete simples e o total de pessoas nesta faixa etária } \\
\text { multiplicado por } 100\end{array}$ & PNUD-Ibge \\
\hline Educação & $\begin{array}{l}\text { Percentual da população de } 6 \text { a } \\
14 \text { anos no ensino fundamental } \\
\text { sem atraso (E_Fund) }\end{array}$ & $\begin{array}{l}\text { Razāo entre o número de pessoas de } 6 \text { a } 14 \text { anos frequentando o ensino } \\
\text { fundamental regular seriado sem atraso idade-série e o número total de } \\
\text { pessoas nessa faixa etária frequentando esse nível de ensino multiplicado } \\
\text { por } 100\end{array}$ & PNUD-Ibge \\
\hline Trabalho & $\begin{array}{l}\text { Grau de formalizaçáo dos } \\
\text { ocupados (Formal) }\end{array}$ & $\begin{array}{l}\text { Razáo entre o número de pessoas de } 18 \text { anos ou mais formalmente } \\
\text { ocupadas e o número total de pessoas ocupadas nessa faixa etária } \\
\text { multiplicado por } 100\end{array}$ & PNUD-Ibge \\
\hline Trabalho & $\begin{array}{l}\text { Taxa de atividade das pessoas } \\
\text { de } 25 \text { a } 29 \text { anos de idade } \\
\text { (Ativ2529) }\end{array}$ & $\begin{array}{l}\text { Razáo entre as pessoas de } 25 \text { a } 29 \text { anos de idade que eram } \\
\text { economicamente ativas, ou seja, que estavam ocupadas ou desocupadas } \\
\text { na semana de referência do Censo e o total de pessoas nesta faixa etária } \\
\text { multiplicado por } 100 \text {. Considera-se desocupada a pessoa que, não } \\
\text { estando ocupada na semana de referência, havia procurado trabalho no } \\
\text { mês anterior à pesquisa }\end{array}$ & PNUD-Ibge \\
\hline Trabalho & $\begin{array}{l}\text { Taxa de desocupação da } \\
\text { populaçã́o de } 18 \text { anos ou mais } \\
\text { de idade (Desoc) }\end{array}$ & $\begin{array}{l}\text { Percentual da populaçáo economicamente ativa (PEA) nessa faixa etária } \\
\text { que estava desocupada, ou seja, que năo estava ocupada na semana } \\
\text { anterior à data do Censo mas havia procurado trabalho ao longo do mês } \\
\text { anterior à data dessa pesquisa }\end{array}$ & PNUD-Ibge \\
\hline
\end{tabular}

Fonte: elaboração própria com dados do Instituto Brasileiro de Geografia e Estatística, Instituto de Pesquisa Econômica Aplicada e Programa das Nações Unidas para o Desenvolvimento, 2010 
Índices de Privação Socioeconômica e de Ruralidade: ferramentas para

o planejamento e pesquisa em Saúde em nível dos municípios brasileiros

Tabela 1.Caracterização das variáveis do estudo sobre medida (Cont.)

\begin{tabular}{|c|c|c|c|}
\hline Dimensão & Variável & Definiçâo & Fonte \\
\hline Trabalho & $\begin{array}{l}\text { Percentual dos ocupados no } \\
\text { setor agropecuário (Agro) }\end{array}$ & $\begin{array}{l}\text { Razáo entre o número de pessoas de } 18 \text { anos ou mais de idade ocupadas } \\
\text { no setor agropecuário e o número total de pessoas ocupadas nessa faixa } \\
\text { etária }\end{array}$ & PNUD-Ibge \\
\hline Habitação & Domicílios adequados (Habit) & $\begin{array}{l}\text { Proporçáo de domićlios com condiçóes mínimas de habitabilidade. } \\
\text { Foram considerados adequados os domicilios que atendessem } \\
\text { simultaneamente os seguintes critérios: densidade de até } 2 \text { moradores por } \\
\text { dormitório; coleta de lixo direta ou indireta por serviço de limpeza; } \\
\text { abastecimento de água por rede geral; e esgotamento sanitário por rede } \\
\text { coletora ou fossa séptica }\end{array}$ & Ibge-Ibge \\
\hline Demografia & $\begin{array}{l}\text { Razáo de dependência } \\
\text { (RazDep) }\end{array}$ & $\begin{array}{l}\text { Razáo entre o número de pessoas com } 14 \text { anos ou menos e de } 65 \text { anos ou } \\
\text { mais de idade (população dependente) e o número de pessoas com idade } \\
\text { de } 15 \text { a } 64 \text { anos (população potencialmente ativa) multiplicado por } 100\end{array}$ & PNUD-Ibge \\
\hline Demografia & $\begin{array}{l}\text { Taxa de urbanizaçáo } \\
\text { (Tax_Urb) }\end{array}$ & Percentagem da populaçáo da área urbana em relaçáo à populaçáo total & $\begin{array}{l}\text { Calculado } \\
\text { PNUD-Ibge }\end{array}$ \\
\hline Demografia & $\begin{array}{l}\text { Percentagem de brancos } \\
\text { (Brancos) }\end{array}$ & Percentagem da população residente de cor/raça branca & Ibge \\
\hline Politicas sociais & $\begin{array}{l}\text { Programas de Assistência } \\
\text { Social (Assis_Soc) }\end{array}$ & $\begin{array}{l}\text { Percetagem da populaçáo residente beneficiada pelo Beneficio de } \\
\text { Prestaçáo Continuada (BPC) para deficientes e idosos, Renda Mensal } \\
\text { Vitalícia (RMV) para deficientes e idosos e/ou Programa Bolsa Família } \\
\text { (PBF) }\end{array}$ & $\begin{array}{l}\text { Calculado } \\
\text { do Ipea e } \\
\text { PNUD-Ibge }\end{array}$ \\
\hline
\end{tabular}

Fonte: elaboração própria com dados do Instituto Brasileiro de Geografia e Estatística, Instituto de Pesquisa Econômica Aplicada e Programa das Nações Unidas para o Desenvolvimento, 2010

Atendidas as suposições estatísticas, aplicou-se a Análise de Componentes Principais, sendo o número de componentes extraídos determinado pela análise do gráfico scree-plot e critério de Kaiser, extraindo-se os componentes que apresentam valores próprios (autovalores ou eigenvalues) superiores a um, explicando a variância de, no mínimo, uma variável. Para a interpretação dos componentes, considerou-se a matriz rotada ortogonalmente pelo método Varimax.

Por fim, na terceira etapa, os índices produzidos foram aplicados para observar seu impacto sobre a saúde, comparando-os com a Esperança de vida por meio da correlação de Spearman. Ademais, foram submetidos à Análise Espacial Exploratória de Áreas através do software R, que, a partir da observação da sua distribuição no espaço, permite testar a plausibilidade dos resultados e simplifica a identificação dos locais em situações mais desfavoráveis, para os quais a prioridade nas políticas públicas deve estar direcionada.

\section{Resultados}

Realizada a consulta da literatura e um processo de exposição-discussão, delineou-se as dimensões do nível contextual dos Determinantes Socioeconômicos da Saúde para os municípios brasileiros, obtendo-se nove grupos: Aspectos econômicos: Educação; Trabalho: Habitação; Demografia; Políticas sociais; Segurança pública; Capital social e Cultura e valores $(1,5,6,7,8)$. Em seguida, as variáveis coletadas nas fontes oficiais foram agrupadas por dimensão e passaram por uma sequência de filtros para a redução da sua quantidade, chegando-se a 13 variáveis para a construção dos índices, conforme ilustrado no fluxograma da figura 1. 


\begin{tabular}{|l|c|}
\hline Filtros: & \multicolumn{1}{|c|}{$\begin{array}{c}\text { Fontes de dados secundários: } \\
\text { IBGE, PNUD e IPEA. }\end{array}$} \\
\hline \multicolumn{1}{|c|}{ Marco teórico } & Mais de 800 variáveis \\
\hline Análise descritiva & 88 variáveis \\
\hline Análise bivariada: Correlação de Spearman & 61 variáveis \\
\hline Marco teórico e Análise de Componentes Principais & 50 variáveis \\
\hline & 13 variáveis \\
\hline
\end{tabular}

Figura 1.Fluxograma do processo de resumo das variáveis socioeconômicas contextuais e construção das Medidas dos Indicadores Socioeconômicos Contextuais. Brasil, 2018 Fonte: elaboração própria

Baseado no modelo teórico construído, primeiro filtro, foram selecionadas 88 variáveis das fontes de dados. Na análise descritiva das variáveis selecionadas para a análise de componentes principais, segundo filtro, apenas a "Proporção de moradias adequadas" apresentou dados indisponíveis, em uma frequência de 4,2\% dos municípios analisados. Dado o baixo percentual de valores perdidos e a importância teórica da variável, sendo a única que representa a dimensão Habitação, manteve-se a variável em questão para as análises seguintes e se fez a estimação dos dados perdidos por meio da técnica de regressão em relação a todas as variáveis selecionadas previamente pelo marco teórico. Neste filtro, foram selecionadas 61 variáveis que apresentaram um maior grau de variabilidade na distribuição de seus valores, bem como mais próximos da normalidade.

No terceiro filtro, a comparação das 61 variáveis entre si e com a esperança de vida, através da correlação de Spearman, foram identificadas 11 variáveis com um número insatisfatório de correlações com coeficiente superiores a 0,3 e menor relação com o indicador de saúde, as quais foram eliminadas das análises subsequentes. No universo de 50 variáveis que permaneceram, foram selecionadas as variáveis mais utilizadas pela literatura e com maior qualidade teórica para explicar a sua respectiva dimensão para a composição dos índices.

Em um processo de exposição-discussão, foram sido realizadas variadas combinações, descartou-se as variáveis que menos atenderam aos critério teóricos e estatísticos, como Renda per capita máxima do $1^{\circ}$ quinto mais pobre, Percentual de indivíduos extremamente pobres, Índice de Theil - L, Produto Interno Bruto e Percentual de moradores em domicílios particulares permanentes com existência de automóvel para uso particular. Ao final, o modelo composto 
pelas 13 variáveis já descritas no quadro 1 resultou como aquele que mais se adequou aos critérios.

Na construção dos índices pela Análise de Componentes Principais, testou-se a aplicabilidade da técnica com as 13 variáveis selecionadas. Como primeiro critério, foi calculada a multicolinearidade, que mede a associação linear entre as variáveis através da matriz de correlação de Spearman. Observou-se uma apropriada multicolinearidade dada a existência de um número substancial de valores de correlação acima de 0,3 , sendo todos significativos ( $p$ $<0,001$ ), bem como a ausência correlações perfeitas (iguais ou maiores a 0,9), o que mostra que não há variáveis que produzem a mesma informação estatística. Ademais, o valor do determinante foi diferente de zero, indicando a presença de, pelo menos, uma dependência linear na matriz.

$\mathrm{Na}$ matriz de correlação anti-imagem, também se confirmou a adequação do conjunto de variáveis introduzidas, pois contém altos valores na sua diagonal principal, indicando que as variáveis selecionadas se correlacionam fortemente entre si, e baixos valores fora dela, mostrando que tais variáveis são pouco influenciadas por outras não contempladas na análise. Além disso, o índice de KMO com valor próximo de $1(0,89)$ indicou uma satisfatória força de correlação do modelo e, examinando a matriz inteira, o teste de Bartlett significativo $(\mathrm{p}<0,001)$ o que assegura a existência de correlações significativas.

Confirmada a aplicabilidade da Análise de Componentes Principais, procedeu-se à extração dos componentes, com vistas a gerar o menor número necessário para explicar o máximo da variância representada pelas variáveis originais. O critério de Kaiser apontou a solução de dois componentes como a mais apropriada, formando duas novas variáveis estatísticas que agruparam os 13 indicadores selecionados e explicaram 71,55\% da variância total dos mesmos. Os componentes foram interpretados conforme a distribuição das cargas fatoriais na matriz rotada, denominando cada componente em função do conjunto de variáveis que obtiveram a maior carga no mesmo.

O primeiro componente formado, que explicou 56,03\% da variância total do modelo, englobou cargas fatoriais altas e negativas das variáveis renda per capita $(-0,75)$, crianças no ensino fundamental sem atraso $(-0,71)$, percentual de brancos $(-0,89)$ e taxa de atividade dos 25 aos 29 anos $(-0,8)$, bem como cargas altas e positivas do índice de Gini $(0,59)$, taxa de analfabetismo $(0,76)$, razão de dependência $(0,71)$, políticas de assistência social $(0,81)$ e taxa de desocupação $(0,73)$. Nesse sentido, tal componente foi denominado Índice de Privação Socioeconômica (IPS). Quanto ao segundo componente, encontramos uma alta e positiva carga de indivíduos ocupados no setor agropecuário $(0,92)$, acompanhado de cargas altas e negativas de taxa de urbanização $(-0,9)$, habitações adequadas $(-0,64)$ e grau de formalização dos ocupados $(-0,7)$, indicando claramente o contexto de Ruralidade, sendo este o nome adotado para o componente em questão (Índice de Ruralidade - IR). 
Uma vez que o Índice de Privação Socioeconômica utilizado pela literatura é construído por uma análise com formação de um único componente e, no presente estudo, na tentativa de geração de um componente único com as 13 variáveis analisadas observou-se que seria produzido um componente pouco robusto (a variância explicada seria de apenas 56\%), executou-se uma segunda Análise de Componentes Principais apenas com as nove variáveis que obtiveram uma maior carga fatorial na Privação Socioeconômica. Ademais, dada a importância observada da Ruralidade para explicar o nível socioeconômico contextual no Brasil, realizou-se uma terceira análise, isolada para as quatro variáveis correspondentes a este componente. Assim, a segunda e a terceira análises formaram os novos Índices de Privação Socioeconômica (63,34\% de variância total explicada) e Ruralidade (73,96\%), respectivamente, cujos valores de comunalidade e carga fatorial estão expressos na tabela 2. Os novos índices atenderam aos critérios de aplicabilidade e agora são independentes entre si, podendo ser replicados e utilizados isoladamente em outros estudos, bem como facilitando a comparação do Índice de Privação Socioeconômica brasileiro com os de outros países.

Tabela 2.Valores de comunalidade e matriz com rotação Varimax das variáveis dos Índices de Privação Socioeconômica e Ruralidade isolados. Brasil, 2018

\begin{tabular}{llllll}
\hline \multicolumn{2}{c}{ Privação Socioeconômica } & \multicolumn{3}{c}{ Ruralidade } \\
\hline Variáveis & Comunalidades & Cargas & Variáveis & Comunalidades & Cargas \\
\hline $\begin{array}{l}\text { Renda per } \\
\text { capita }\end{array}$ & 0,78 & $-0,88$ & $\begin{array}{l}\text { Formalização } \\
\text { dos ocupados }\end{array}$ & 0,75 & $-0,86$ \\
\hline Índice Gini & 0,35 & 0,59 & $\begin{array}{l}\text { Setor } \\
\text { Agropecuário }\end{array}$ & 0,79 & 0,89 \\
\hline Analfabetismo & 0,78 & 0,88 & $\begin{array}{l}\text { Habitaçóes } \\
\text { adequadas }\end{array}$ & 0,63 & $-0,79$ \\
\hline $\begin{array}{l}\text { Ens. } \\
\text { Fundamental } \\
\text { sem atraso }\end{array}$ & 0,57 & $-0,76$ & $\begin{array}{l}\text { Taxa de } \\
\text { urbanização }\end{array}$ & 0,79 & $-0,89$ \\
\hline $\begin{array}{l}\text { Atividade dos } \\
\text { 25 a 29 anos }\end{array}$ & 0,69 & & & & \\
\hline Desocupação & 0,29 & $-0,83$ & & & \\
\hline $\begin{array}{l}\text { Razão de } \\
\text { Dependência }\end{array}$ & 0,69 & 0,54 & & & \\
\hline $\begin{array}{l}\text { Percentual de } \\
\text { brancos }\end{array}$ & 0,76 & 0,83 & & & \\
\hline $\begin{array}{l}\text { Assistência } \\
\text { Social }\end{array}$ & 0,8 & $-0,87$ & & & \\
\hline
\end{tabular}

Fonte: elaboração própria com dados do Instituto Brasileiro de Geografia e Estatística, Instituto de Pesquisa Econômica Aplicada e Programa das Nações Unidas para o Desenvolvimento, 2010 
Observa-se um alto percentual de variância explicada dos indicadores pelo IPS e IR, com exceção do índice de Gini e da Taxa de desocupação, que obtiveram um valor de comunalidade inferior a 0,5 . Apesar do valor baixo, os mesmos foram mantidos no modelo devido a sua importância teórica para as iniquidades em saúde e porque os outros indicadores de compatíveis disponíveis, que poderiam substituí-lo, não obtiveram maior ajuste ao modelo.

Os índices produzidos variam de valores negativos a positivos, com valor mínimo de -2,31 em Westfália/RS e máximo de 2,91 em Amajari/RR (maior desvantagem socioeconômica) para o Índice de Privação Socioeconômica e mínimo de -2,24 em Dois Irmãos/RS e máximo de 2,22 (maior ruralidade) em Coronel Pilar/RS para o Índice de Ruralidade. A distribuição dos dados, observada a partir de histogramas, é bastante diferente entre os índices, tendo a Ruralidade uma distribuição mais próxima à normal, indicando que a maioria dos municípios tem valores em torno da média do Índice de Ruralidade, com poucos casos extremos, enquanto a Privação Socioeconômica apresenta duas modas.

Esta característica bimodal dos dados do Índice de Privação Socioeconômica expõe a presença de duas diferentes subpopulações de municípios no Brasil: os de maior vantagem socioeconômica (de valores negativos) e os de maior desvantagem (de valores positivos). A divisão do país em duas diferentes populações é também observada na terceira etapa do estudo, ao se fazer a aplicação prática dos índices através da Análise espacial de áreas, apresentadas nas figuras 2 (Índice de Privação) e 3 (Índice de Ruralidade).

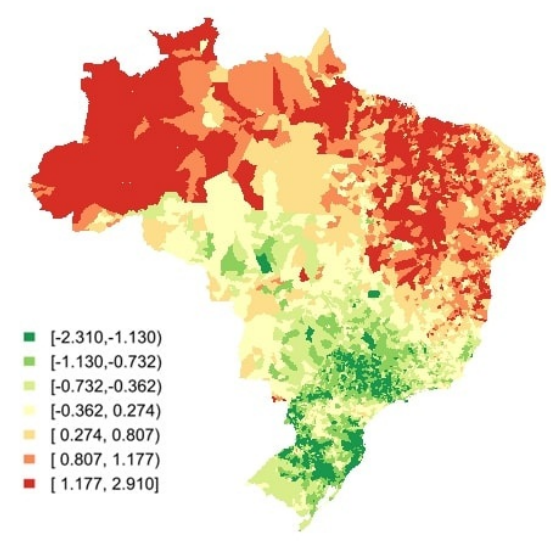

Figura 2. Distribuição espacial do Índice de Privação Socioeconômica. Brasil, 2018 Fonte: elaboração própria

O mapa do Índice de Privação Socioeconômica revela claramente as iniquidades presentes no Brasil, dividindo o território brasileiro em dois: porção norte com maior privação socioeconômica e porção centro-sul de maior desenvolvimento. Identificou-se que $91,1 \%$ dos municípios da região Norte e $98,2 \%$ da região Nordeste detêm valores positivos de privação 
socioeconômica, enquanto na porção do país de maior desenvolvimento tal característica está presente em apenas 22,2\% dos municípios do Sudeste, 24\% do Centro-Oeste e 1,4\% do Sul.

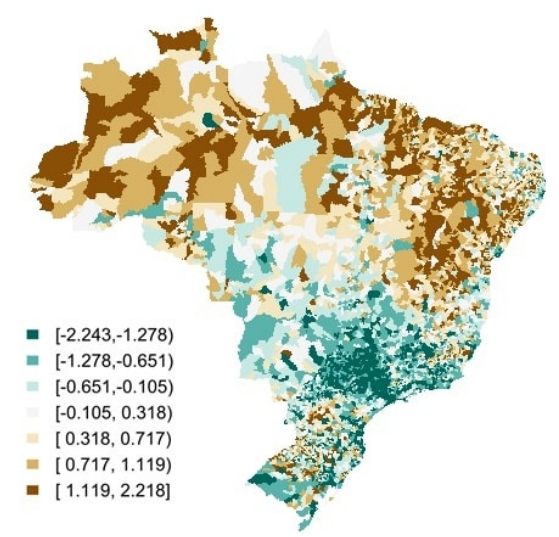

Figura 3.Distribuição espacial do Índice de Ruralidade. Brasil, 2018

Fonte: elaboração própria

O Índice de Ruralidade apresenta padrão mais heterogêneo, substancialmente presente em todas as regiões do Brasil, com exceção do Sudeste que é pouco rural (24,3\% dos municípios com Índice de Ruralidade positivo) e representa 49,2\% do grupo de municípios brasileiros mais urbanizados (Ruralidade negativa). As regiões Sul e Centro-Oeste também são mais urbanizadas em sua maioria, com 52\% e 56\% municípios, respectivamente, com Ruralidade negativa. Já o Norte e Nordeste são regiões predominantemente mais ruralizadas, com $81,7 \%$ e $80,9 \%$ dos municípios, respectivamente, no grupo dos municípios brasileiros com Ruralidade positiva.

Aplicando-se os índices em comparação à Esperança de vida, por meio da correlação de Spearman, temos que as mesmas estão significativamente correlacionadas com este indicador de saúde $(p<0,001)$. O Índice de Privação Socioeconômica representa o componente mais relacionado à saúde, com um alto e negativo valor de correlação $(-0,83)$ e explicando 68,9\% da variabilidade da Esperança de vida. Já o Índice de Ruralidade, detém um valor moderado e negativo (-0,62), com poder de explicação de 38,4\% da mesma variabilidade. Portanto, inferese que maiores níveis de Privação Socioeconômica e Ruralidade determinam prejuízos à saúde das populações.

A fim de observarmos a interação dos dois componentes, fez-se o agrupamento dos 5565 municípios a partir da categorização em duas classes dos Índices de Privação Socioeconômica (Maior Privação Socioeconômica os municípios com valores positivos e Menor Privação Socioeconômica aqueles com valores negativos) e de Ruralidade (Ruralizados os municípios com valores positivos de Ruralidade e Urbanizados aqueles com valores negativos). Assim, 
os municípios brasileiros se agruparam em Maior Privação Socioeconômica e Ruralizados (2149 municípios), Maior Privação Socioeconômica e Urbanizados (521), Menor Privação Socioeconômica e Ruralizados (849) e Menor Privação Socioeconômica e Urbanizados (2046).

A partir desta categorização é destacada a importância que a Ruralidade tem por sua relação com a Privação Socioeconômica no território brasileiro, pois $80,5 \%$ dos municípios que foram classificados como de Maior Privação Socioeconômica São Ruralizados. O grupo de municípios de Maior Privação Socioeconômica e Ruralizados chama atenção também na sua relação com a Esperança de vida, observando-se a maior influência que o Índice de Privação Socioeconômica tem sobre a saúde, quando comparado ao Índice de Ruralidade, uma vez que os menores valores de Esperança de vida dizem respeito aos dois grupos de municípios com a característica de maior Privação Socioeconômica. No entanto, a Ruralidade tem sua importância destacada por complementar a informação dada pela Privação Socioeconômica, demonstrando diferenças de níveis de saúde dentro das classes deste, nas quais os 2149 municípios de Maior Privação Socioeconômica e Ruralizados correspondem àqueles com menor esperança de vida (média de 70,7 anos) e, portanto, para os quais deve estar direcionada a prioridade na execução de políticas públicas.

Tendo em vista que os índices criados têm o objetivo de subsidiar políticas públicas e execução de pesquisas epidemiológicas, os dados produzidos estão disponíveis em uma plataforma online pública para que todos tenham acesso livre e possam aplicá-1o ${ }^{[1]}$.

\section{Discussao}

As duas Medidas dos determinantes contextuais formadas, Índice de Privação Socioeconômica e de Ruralidade, resumiram o grande número de variáveis correspondentes ao nível dos DSS em questão, traduzindo a vulnerabilidade socioeconômica dos municípios brasileiros de modo evidente. Por seu potencial efeito negativo em saúde, tais fatores devem considerados para a compreensão dos mecanismos envolvidos com as doenças e agravos à saúde e, por conseguinte, para o planejamento de macropolíticas públicas que intervenham nas iniquidades em saúde, a partir da informação dos municípios com maior necessidade de atenção.

Os índices são utilizados com frequência para a compreensão de eventos que não apresentam uma medida simples ou direta, como ocorre com o constructo multidimensional socioeconômico contextual, que tem sido trabalhado pela literatura com índices de privação $(14,15,16,17)$. Em seu conceito, o Índice de Privação Socioeconômica não significa apenas pobreza, apesar de estar relacionado com a mesma. Enquanto este representa a escassez de recursos financeiros, a Privação Socioeconômica, determinada de modo agregado pela situação de vulnerabilidade da população residente no território, constitui uma medida mais global, com escassez de recursos de variados tipos, como de educação, condições adequadas de habitação e, inclusive, a renda (15).

De forma clara, a Privação Socioeconômica dividiu o Brasil em dois grandes grupos: municípios de maior desvantagem socioeconômica concentrados nas regiões Norte e Nordeste e os de maior 
vantagem no Centro-Oeste e, especialmente, Sul e Sudeste. Tal polarização é histórica e já conhecida, na qual as regiões Sul e Sudeste detêm um maior aporte da atividade industrial do país, enquanto o Norte e Nordeste se desenvolvem de modo mais retardatário no processo econômico brasileiro (18).

Em comparação a outros estudos $(14,15,16,17)$, a análise no contexto brasileiro trouxe uma novidade: a formação de um segundo componente, a Ruralidade. Junto à Privação Socioeconômica, a Ruralidade auxilia na compreensão das iniquidades em saúde de modo complementar à primeira, que se mostrou mais importante na discriminação dos municípios. Apesar de se ter optado pela construção de dois índices independentes por meio da segunda Análise de Componentes Principais, recomenda-se para os estudos que venham a utilizar os índices que, para uma compreensão mais aprofundada acerca dos determinantes contextuais em municípios do Brasil, o Índice de Privação Socioeconômica não seja considerado isoladamente, mas acompanhado do Índice de Ruralidade.

A zona de residência (rural ou urbana) já tem sido destaca nos últimos anos como um importante determinante da saúde (19). Tal importância foi confirmada no presente estudo, no qual surgiu o componente Ruralidade como determinante contextual sem qualquer indução. A Ruralidade expôs diferenças importantes dentro das classes de Privação Socioeconômica, sendo os de maior Privação e Ruralizados $(78,6 \%$ do Norte e $80,8 \%$ do Nordeste) aqueles municípios com maior desvantagem e os de menor Privação e Urbanizados (68,5\% do Sudeste, 51,8\% do Sul e $50 \%$ do Centro-Oeste) aqueles com maior vantagem quando da comparação com o indicador de saúde. A menor exposição à poluição e atividades de trabalho com menor carga de estresse trazem benefícios à saúde dos indivíduos que residem em zonas rurais. Por outro lado, a ruralidade também está caracterizada por uma baixa concentração de habitantes, grandes distâncias, estradas precárias e baixa oferta de serviços, o que culmina em um menor acesso à atenção à saúde, seja ela pública ou privada, fatores estes que prevaleceram como influência negativa da Ruralidade sobre a Saúde no Brasil (20).

Vale destacar que a ruralidade no Norte e Nordeste, grupo de maior privação socioeconômica, tem aspectos distintos do centro-sul do país, de menor privação socioeconômica. Enquanto estes realizam atividades com tecnologias altamente modernas, o Norte e Nordeste ainda possuem práticas mais rudimentares e precárias (21). Adiciona-se a isso as diferenças nos entornos rurais presentes nos dois grupos, sendo as redes urbanas mais desenvolvidas nos municípios de menor privação socioeconômica, ofertando um leque de serviços mais completo e, geralmente, com maior qualidade.

Além de auxiliar os gestores de políticas públicas para o enfrentamento das iniquidades em saúde e diante da dificuldade de se executar análises e interpretações com o grande leque de dados disponíveis, bem como de se selecionar as variáveis mais adequadas, os índices produzidos contribuem para as pesquisas em saúde por serem dados sintetizados que 
representam globalmente o constructo dos determinantes contextuais, podendo ser trabalhados isoladamente. Assim, os índices podem ser utilizados para: a) analisar o efeito contextual dos fatores socioeconômicos de municípios sobre a saúde, porém sem determinar relações causais; b) utilização dos determinantes contextuais como uma variável de confusão que se deseja controlar; e c) quando não há razão teórica para a análise com um indicador simples em detrimento de um índice (14).

Em relação aos índices socioeconômicos contextuais já utilizados no Brasil, como o IDH e IFDM, os índices aqui produzidos apresentam vantagens quando da aplicação nas pesquisas em saúde, pois agregam um aporte teórico mais aprofundado acerca dos modelos dos DSS. Ademais, o IDH e o IFDM contêm dados de saúde na sua composição, distorcendo a medida socioeconômica. No entanto, os índices apresentam limitações que devem ser consideradas. Primeiramente, há uma limitação relacionada ao tipo de estudo (ecológico), que não permite fazer uma inferência causal direta sobre fenômenos individuais de achados encontrados no nível agregado, viés este conhecido como falácia ecológica.

Para minimizar o viés ecológico, deve-se utilizar dados agrupados nas menores unidades de análise possível, pois é mais provável que a população seja mais homogênea e, assim, melhor se detecte as diferenças em saúde $(14,22)$. Neste sentido, foi utilizada a menor unidade territorial disponível nas fontes de dados socioeconômicos secundários: os municípios. Ademais, tendo em vista que se pretende fornecer subsídios para o planejamento em saúde, a escolha do nível municipal se justifica pela sua governabilidade, já que corresponde a esfera de governo mais próximo aos indivíduos. Como desvantagem, temos a grande amplitude do tamanho populacional dos municípios brasileiros, variando de 805 no município de Borá/SP a 11.253.503 habitantes em São Paulo/SP, segundo censo Ibge 2010 (10), determinando diferenças no grau de homogeneidade da população.

O censo é a fonte ideal para a análise de indicadores socioeconômicos, pois produz dados reais, e não estimativas. Porém, seus dados são disponibilizados com uma frequência apenas decenal e com um número pouco extenso de variáveis coletadas devido ao seu alto custo. Neste sentido, foram utilizados também dados da Pesquisa Nacional por Amostra de Domicílios (PNAD) realizada pelo mesmo instituto de pesquisa (Ibge) e dados de outras fontes (Ipea e PNUD), para a obtenção de índices que representassem o nível socioeconômico contextual com a maior completude teórica possível.

Ainda assim, dentre as nove dimensões delimitadas, a Segurança Pública e Capital Social não puderam ser contemplados devido à ausência de indicadores nas fontes oficiais. Ademais, foi encontrada apenas uma variável para medir Cultura e valores. Também constitui uma limitação o total de 4,2\% de municípios com valores ausentes para a variável "Proporção de moradias adequadas", destacando a necessidade de melhorias no processo de coleta e disponibilização dos dados. 
Diferentemente de estudos em outros países $(14,15,16,17)$, a desocupação, apontada como um dos principais determinantes das iniquidades em saúde, manifestou um menor ajuste ao modelo do índice de privação socioeconômica para os municípios brasileiros. Este achado pode ser explicado pela geração de indivíduos em idade economicamente ativa que não estudam, nem trabalham, popularmente chamada no Brasil como geração "nem-nem", que segundo dados da PNAD em 2012 correspondeu a quase um quarto $(23,4 \%)$ dos jovens de 18 a 24 anos de idade (23). Sendo assim, muitos dos jovens "nem-nem" não são considerados no cálculo da taxa de desocupação porque não estavam procurando por trabalho, o que ameniza a taxa de desocupação e seu efeito na privação socioeconômica.

Quanto ao Índice de Gini, que obteve o menor efeito dentre as variáveis que compõem o Índice de Privação Socioeconômica, seu menor ajuste reflete que a desigualdade de renda não é uma característica preponderante entre os municípios com alta Privação Socioeconômica, os quais detêm menor renda per capita. Nesse contexto, a "igualdade" se torna um fator negativo, pois significa que a população de tais municípios é "igualmente” de baixa renda. Pode-se dizer, então, que a "igualdade" neste caso não serve por implicar em uma situação de pobreza generalizada e uma maior dependência do Estado.

Outra variável que chama a atenção no modelo proposto é o Percentual de brancos na composição do Índice de Privação Socioeconômica, expressando as iniquidades sociais, já que se trata de uma variável que per si deveria trazer apenas uma informação demográfica, a cor da pele. Tal situação de iniquidade pela cor da pele já é conhecida, sendo apontado por Henriques, em 2001 (24), que "nascer de cor parda ou de cor preta aumenta de forma significativa a probabilidade de um brasileiro ser pobre", porém, é chocante observar que o impacto da cor da pele sobre as iniquidades sociais se estende de forma tão clara ao nível ecológico, representando um importante fator de categorização dos municípios de acordo com a privação socioeconômica, dado que esta correspondeu à variável que obteve o maior ajuste ao modelo estatístico, caracterizado pela maior comunalidade e carga fatorial.

Apesar de se ter um grande número de variáveis disponíveis, não foi possível contemplar todas as dimensões do nível estudado devido à ausência de variáveis correspondentes às dimensões de segurança pública e capital social e poucas de cultura e valores nas fontes de dados públicas e oficiais. Tais dimensões constituem uma dificuldade também em estudos em outros países, fazendo-se necessário o desenvolvimento de modos de medi-las (25). Para as dimensões segurança pública e capital social, alguns trabalhos utilizam as taxas de homicídios e suicídio para medi-las, respectivamente. Entretanto, estas varáveis representam questões de saúde que se posicionam como evento de interesse (desfecho) em investigações na área da saúde e não como variável independente, bem como incluí-las juntas com variáveis específicas dos fatores socioeconômicos contextuais para a construção de um índice pode levar distorções no mesmo. 


\section{Conclusao}

O presente estudo resumiu o universo de indicadores do nível socioeconômico contextual para os municípios do Brasil e traduziu a iniquidade na distribuição dos recursos e, por conseguinte, na saúde em dois índices: Privação Socioeconômica e Ruralidade. A partir da comparação com o indicador de saúde utilizado, Esperança de vida, observou-se que o componente de maior efeito sobre a saúde é a Privação Socioeconômica, no entanto, a ruralidade representa um importante fator no contexto brasileiro e, diferentemente do que foi observado em outros estudos, complementa a Privação Socioeconômica, destacando importantes diferenças nas classes desta e devendo acompanhá-la nas análises em saúde. Para um planejamento de políticas públicas eficazes, deve-se consolidar a priorização dos 2149 municípios identificados como de maior Privação Socioeconômica e Ruralidade, que representam o grupo de maior vulnerabilidade em saúde, localizados especialmente no Norte e Nordeste.

Para os próximos estudos, recomenda-se a análise dos índices em relação aos diferentes problemas de saúde, como variável independente principal, de confusão ou modificadora de efeito, a fim de melhor compreender os mecanismos envolvidos e contribuir para que o planejamento de políticas públicas atenda às reais demandas. Recomenda-se também a coleta e disponibilização de dados que meçam as dimensões do nível socioeconômico contextual não contempladas pelos índices: a segurança pública, o capital social e a cultura e valores, permitindo um aprimoramento de tais medidas de modo a atender mais fielmente aos marcos conceituais, explorando o nível socioeconômico contextual por inteiro.

\section{Agradecimentos}

À Coordenação de Aperfeiçoamento de Pessoal de Nível superior (Capes), pelo financiamento deste projeto através do Programa de Doutorado Sanduíche no Exterior (PDSE).

\section{Referências}

1. Dalghren G, Whitehead M. Policies and strategies to promote social equity in health. Estocolmo: Institute for Future Studies; 2007.

2. Carvalho AI. A saúde no Brasil em 2030 - Prospecção estratégica do sistema de saúde brasileiro: população e perfil sanitário. Rio de Janeiro: Fiocruz/Ipea/Ministério da Saúde/Secretaria de Assuntos Estratégicos da Presidência da República; 2013 [citado em 2016 nov. 07]. Determinantes Sociais, Econômicos e Ambientais da Saúde; p. 43-56. Disponível em: https://www.saudeamanha.fiocruz.br /wp-content/uploads/2016/07/saude-2030livro_0.pdf

3. Organização Mundial da Saúde. Constituição [Internet]. 1946 [citado em 2016 nov. 07]. Disponível em: https://www.direitoshumanos.usp.br/index.php/OMS-Organiza\%C3\%A7\%C3\%A3o-Mundialda-Sa\%C3\%BAde/constituicao-da-organizacao-mundial-da-saude-omswho.html

4. Guerra G, Borde E, Salgado-de-Snyder N, VNS. Measuring health inequities in low and middle income countries for the development of observatories on inequities and social determinants of health. Int $\mathrm{J}$ Equity Health. 2016;15(9):1-10. 
Tamires Carneiro de Oliveira Mendes/ Marc Marí-Dell’Olmo/ Albert Pratsb Uribe/ Kenio Costa Lima/ et ai.

5. Borrell C, Malmusi D. La investigación sobre los determinantes sociales y las desigualdades em salud: evidencias para la salud eb todas las políticas. Informe Sespas 2010. Gac Sanit. 2010;24(sup 1):101-108.

6. Borrell C, Pons-Vigués M, Morrison J, Díez È. Factors and processes influencing health inequalities in urban areas. J Epidemiol Community Health. 2013;67(5):389-391.

7. Solar O, Irwin A. A conceptual framework for action on the social determinants of health. Comission on Social Determinantes of Health. Ginebra: WHO; 2007.

8. Comisión para reducir las desigualdades sociales en salud en España. Propuesta de políticas e intervenciones para reducir las desigualdades sociales en salud en España. Gacet. Sanit. 2012;26(2):182-189.

9. Programa das Nações Unidas para o Desenvolvimento (PNUD). Atlas do Desenvolvimento Humano no Brasil [Internet]. 2015. Disponível em: https://www.atlasbrasil.org.br/2013

10. Instituto Brasileiro de Geografia e Estatística (Ibge) [Site]. Brasília: Ibge; 2015. Disponível em: h ttps://www.ibge.gov.br/home/

11. Brasil, Instituto de Pesquisa Econcômica Aplicada (Ipea) [Site]. Brasília: Ipea; 2015. Disponível em: https://www.ipeadata.gov.br/

12. Carr-hill R, Chalmers-dixon P. The Public Health Observatory Handook of Health Inequalities Measurement. Londres: South East Public Health Observatory; 2005.

13. Fávero LP, Belfiore P, Silva FL, Chan BL. Análise de dados: modelagem multivariada para tomada de decisões. Rio de Janeiro: Elsevier; 2009.

14. Domínguez-Berjón MF, Borrell C, Cano-Serral G, Esnaola S, Nolasco A, Passarín MI, et al. Construcción de un índice de deprivación a partir de datos censales en grandes ciudades españolas (Proyecto Medea). Gac Sanit. 2008;22(3):179-187.

15. Smith T, Noble M, Noble S, Wright G, Mclennan D, Plunkett E. The English Indices of Deprivation 2015. Technical Report. Londres: Department for Communities and Local Government; 2015.

16. Pampalon R, Hamel D, Gamache P, Raymond G. A deprivation index for health planning in Canada. Chronic Dis. Can. 2009;19(4):178-191.

17. Ocaña-Riola R, Saurina V, Fernández-Ajuria A, Lertxundi A, Sánchez-Cantalejo C, Saez M, et al. Area deprivation and mortality in the provincial capital cities of Andalusia and Catalonia (Spain). J Epidemiol Community Health. 2008;62:147-152.

18. Araújo JA, Morais GAS. Desigualdade de renda e sua decomposição no Brasil e nas regiões brasileiras. Rev. Econ. NE. 2014;45(4):35-51.

19. Santana P, Costa C, Marí-Dell'Olmo M, Gotsens M, Borrell C. Mortality, material deprivation and urbanization: exploring the social patterns of a metropolitan area. Int J Equity Health. 2015;14:55. h ttps://www.doi.org/10.1186/s12939-015-0182-y 
Índices de Privação Socioeconômica e de Ruralidade: ferramentas para

o planejamento e pesquisa em Saúde em nível dos municípios brasileiros

20. Tavares DMS, Arduini GO, Martins NPF, Dias FA, Ferreira LA. Características socioeconômicas e qualidade de vida de idosos urbanos e rurais com doenças cardíacas. Rev Gaúcha Enferm. 2015;36(3):21-27. http://dx.doi.org/10.1590/1983-1447.2015.03.45470

21. Nascimento CA. A pluriatividade das famílias rurais no Nordeste e no Sul do Brasil: pobreza rural e políticas públicas. Econ. Soc. 2009;18(2):317-348.

22. Medronho RA, Bloch KV, Ronir RL, Werneck GL. Epidemiologia. 2.a ed. Rio de Janeiro: Atheneu; 2009.

23. Instituto Brasileiro de Geografia e Estatística (Ibge). Síntese de Indicadores Sociais. Uma análise das condições de vida da população brasileira 2013. Diretoria de Pesquisas. Coordenação de População e Indicadores Sociais [Internet]. Rio de Janeiro: Ibge, 2013. Disponível em: https://www.biblioteca .ibge.gov.br/visualizacao/livros/liv66777.pdf

24. Henriques R. Desigualdade racial no Brasil: evolução das condições de vida na década de 90. Brasília: Instituto de Pesquisa Econômica Aplicada; 2001; p. 1-52.

25. Ferreira MAF, Latorres MRDO. Desigualdade social e os estudos epidemiológicos: uma reflexão. Ciênc. Saúde Coletiva 2012;17(9):2525-2531.

Notas

[1] As cartografias e dados para todos os municípios podem ser visualizados e ser feito o download no endereço eletrônico: https://w ww.public.tableau.com/profile/albert.p.\#!/vizhome/Privaao/Panel1

* $\quad$ Artigo de pesquisa Este estudo é resultado do Programa de Doutorado Sanduíche no Exterior (PDSE) realizado na Agència de Salut Pública de Barcelona (Cataluña, Espanha), financiando pela Coordenação de Aperfeiçoamento de Pessoal de Nível superior (Capes). 